\section{TargeTALE: A Web Resource to Identify TALEs in Xanthomonas Genomes and Their Respective Targets}

\author{
Frederico Schmitt Kremer, ${ }^{\dagger}$ Amanda Munari Guimarães, Christian Domingues Sanchez, \\ and Luciano da Silva Pinto ${ }^{\dagger}$
}

Centro de Desenvolvimento Tecnológico, Universidade Federal de Pelotas, Capão do Leão, Rio Grande do Sul, Brazil

\begin{abstract}
The Xanthomonas genus, comprises more than 30 species of gram-negative bacteria, most of which are pathogens of plants with high economic value, such as rice, common bean, and maize. Transcription activator-like effectors (TALEs), which act by regulating the host gene expression, are some of the major virulence factors of these bacteria. We present a novel tool to identify TALE genes in the genome of Xanthomonas strains and their respective targets. The analysis of the results obtained by TargeTALE in a proof-of-concept validation demonstrate that, at optimum setting, approximately $93 \%$ of the predicted target genes with available expression data were confirmed as upregulated during the infection, indicating that the tool might be useful for researchers in the field.
\end{abstract}

Genus Xanthomonas includes more than 30 species of gram-negative bacteria, most of which are phytopathogenic and infect plants of high economic importance (Vauterin et al. 2000). X. oryzae, for example, is a major threat to rice (Oryza sativa) production, and its pathovars, $X$. oryzae pv. oryzae and $X$. oryzae pv. oryzicola, which are etiological agents of bacterial blight and bacterial leaf streak, respectively, have re-emerged as worldwide concerns during the past decades. $X$. oryzae pv. oryzae has already been reported to be capable of reducing production yield by up to $80 \%$ in susceptible regions, while $X$. oryzae pv. oryzicola has been reported to cause losses of up to $30 \%$ during epidemics in certain regions (Niño-Liu et al. 2006). In a similar way, other species of the genus, such as $X$. citri, which infects many species of the Citrus genus, $X$. citri subsp. fuscans, which infects many species of beans, and $X$. axonopodis pv. glycines, which infects soybean (Glycine max), also have a drastic impact on productivity, depending on the geographic region, and lead to many economic losses as well.

Transcription activation-like effectors (TALEs) are proteins produced by several Xanthomonas species and are secreted via the type III secretion system. These proteins act as modulators of gene expression in the host cells by specifically recognizing promoter regions in the host genome. The promoter sequence is recognized by variable domains comprising repeats of approximately 34 amino acids with two hypervariable residues, which are called repeat variable di-residues (RVDs) (Muñoz Bodnar et al. 2013).

${ }^{\dagger}$ Corresponding authors: L. da Silva Pinto Is_pinto@ @otmail.com and F. Kremer; fred.s.kremer@gmail.com

*The $e$-Xtra logo stands for "electronic extra" and indicates that two supplementary tables are published online.

The author(s) declare no conflict of interest.

Accepted for publication 15 August 2019.

(C) 2019 The American Phytopathological Society

\section{Funding}

This work was supported by Conselho Nacional de Desenvolvimento Científico e Tecnológico (CNPq, Brazil).

\section{Keywords}

bioinformatics, effector proteins, genome annotation, phytopathogens, T3SS 
A previously described model of the relationship between the RVD sequence and DNA specificity (Boch et al. 2009; Moscou and Bogdanove 2009) has made it possible to predict the targeted regions of a certain TALE in a DNA sequence in silico, based on the analysis of its RVDs. In fact, some tools, such as TargetFinder (Doyle et al. 2012), StoryTeller (PérezQuintero et al. 2013), TALgetter (Grau et al. 2013), TALvez (Pérez-Quintero et al. 2013), AnnoTALE (Grau et al. 2016), and daTALbase (Pérez-Quintero et al. 2018), have already been developed for such analysis. These tools usually allow the identification and prediction of targets based on a provided set of sequences or classification of TALEs, but researchers with nonbioinformatics backgrounds might find it difficult to use them for genome-wide analysis of TALEs and their respective targets in newly sequenced genomes. In addition, although TALvez provides a web-based interface and databases from various plant hosts, it requires the input of previously annotated RVD sequences and does not accept unannotated genomes in FASTA format, which makes it largely inapplicable for processing newly sequenced genomes. Furthermore, when preloaded databases are used, the TALvez output is also mainly limited to previously described promoters, which makes the identification of novel target genes difficult. DaTALbase, also a web-based tool, provides integrated datasets of gene expression during infection along with TALE target prediction and already identified TALEs but lacks TALE identification from new genomes and is currently limited to four hosts (rice, cassava, barley, and cabbage).

In order to provide a simple tool for Xanthomonas researchers to analyze TALE genes, we have developed a new pipeline named TargeTALE, which integrates TALE predictors, a wide variety of promoter datasets, TALE target predictors, and functional annotations of the identified targets using Gene Ontology (GO) terms.

TargeTALE was developed using the programming language Python and the third-party packages BioPython, Bioservices, and Requests and was deployed as a webserver running on top of Apache HTTP server. TALE genes are predicted using AnnoTALE (Grau et al. 2016), which is also used to assign a standardized class name to TALE genes and identify the RVD region. Promoter databases were retrieved for a wide variety of model and nonmodel species from different sources, including Ensembl, the Joint Genome Institute, The Arabidopsis Information Resource (TAIR), and Osiris (Morris et al. 2008).

The targets of each TALE are identified using TALgetter (Grau et al. 2013), and only the hits with $P$ value $<0.05$ and $E$ value $<1 \mathrm{e}-10$ are selected. Finally, the Uniprot ID of each target is accessed from the Uniprot database, using its client implemented in the Python Bioservices package. The Uniprot ID is used to retrieve its GO annotation from QuickGO (Binns et al. 2009).

As a proof-of-concept to evaluate the reliability of the pipeline, we analyzed six genomes of X. oryzae: X. oryzae pv. oryzae PXO99A (GenBank CP000967.2), X. oryzae pv. oryzae PXO86 (GenBank CP007166.1), X. oryzae pv. oryzicola CFBP7331 (GenBank CP011958.1), X. oryzae pv. oryzicola B8-12 (GenBank CP011955.1), X. oryzae pv. oryzicola BLS256 (GenBank CP003057.2), and X. oryzae pv. oryzicola CFBP7342 (GenBank CP007221.1), using the different promoterome datasets of $O$. sativa derived from Osiris (250, $500,750,1,000,1,500,2,000$, and 3,000 bp upstream, considering gene models or transcript alignment). Then, the identified target genes in each combination of strain and dataset were matched with strain-specific gene expression data available in the European Bioinformatics Institute (EBI) Expression Atlas database. The experiment E-GEOD-36272 was used for $X$. oryzae pv. oryzae strains, while E-GEOD-67588 was used for $X$. oryzae pv. oryzicola.

In the first analysis, we considered as positive hits those genes whose expression levels were increased during the infection by the specific strains and as negative those whose expression was not. As not all genes indexed in the promoterome are covered by the gene sets used in the expression studies, we also calculated the percentage of correct hits considering only those genes with data available in the expression datasets. Genes were considered upregulated during the infection if the $\log _{2}$ fold change is $\geq 2$.

The pipeline was implemented as a webserver and does not require any registration to be used. During the submission, the user must only upload a FASTA file containing the genome to be analyzed, select one of the available promoterome datasets, and provide his e-mail address, and, when the job is finished, the user will receive an e-mail with a link to access the analysis report. The results might also be downloaded as a ".tar.gz" file, which includes tables, in tab-separated value format, and, with a summary of the TALEs and targets identified, GO annotations of the targets and the intermediary results of each program.

1578 / Molecular Plant-Microbe Interactions 
The results of the evaluation of the tool, using gene expression data of $O$. sativa after infection by different strains of $X$. oryzae, are presented in Supplementary Table S1, which presents data on the percentage of targets identified as upregulated excluding those targets not indexed in the respective study, and Supplementary Table S2, which includes those targets not indexed in the respective study.

Currently, TargeTALE provides promoter datasets for 16 different plant species, covering common hosts of Xanthomonas spp., such as rice ( $O$. sativa), common bean (Phaseolus vulgaris), sweet orange (Citrus sinensis), tomato (Solanum lycopersicum), soybean (Glycine max), and maize (Zea mays), and model organisms to study hostpathogen interactions and pathogenesis, such as Arabidopsis thaliana (Buell 2002) and Brachypodium distachyon (Fitzgerald et al. 2015). For each host, we also provide datasets of promoter sequences derived from different upstream distances from its genes. In the case of datasets generated using in-house scripts and the rice datasets derived from Osiris, we provide upstream sequences of $250,500,750$, and 1,000 bp. Finally, datasets for A. thaliana were obtained from TAIR (Rhee et al. 2003) and are available for upstream distances of 500 and 1,000 bp.

In the validation of our pipeline using $X$. oryzae data, when only the identified target genes having gene expression data in the EBI Gene Expression Atlas for the specified strain were considered, we were able to obtain a mean confirmation rate of approximately $95 \%$, using the 250 bp upstream derived from genes identified based on transcript datasets; a slight difference was observed from strain to strain and when considering different upstream distances. The higher accuracy with 250-bp datasets was also observed when considering the ratios of genes confirmed to be upregulated during the infection versus when the full set of predicted target genes were considered, although this value dropped to $35.9 \%$. However, it is unclear whether this was a consequence of discrepancy in the gene expression datasets, although previous studies have already demonstrated that the effective activation of a target gene expression may require additional factors, such as the presence of multiple TALEs binding to the same gene, even in the opposite DNA strand (Streubel et al. 2017). Additionally, it was already reported that TALEs usually bind in positions located $300 \mathrm{bp}$ around the transcription start site (Grau et al. 2013), which might explain why the 250-bp upstream datasets led to more accurate results and why better results were usually obtained when using data derived from transcripts and not from predicted gene models. Thus, the analysis also indicated that datasets of longer upstream regions do not provide better results, as they might increase the occurrence of falsepositives.

When compared with other web-based tools, such as TALVEZ and daTALbase, TargeTALE might provide a more straightforward way to analyze newly sequenced genomes from Xanthomonas spp. by combining a web-based interface and methods for TALE prediction, target identification, and functional annotation; while these tools require already defined RVD sequences, they don't provide TALE identification. It also might be more userfriendly than AnnoTALE and TALgetter while keeping most of their main features and extending the results with the data from GO annotation.

We strongly discourage the submission of draft genomes sequenced using short-read platforms such as Illumina MiSeq and Ion Torrent PGM, as the repetitive structure of TALE genes may lead to mis-assemblies in these cases, so long-read sequenced genomes (Peng et al. 2016), obtained from platforms such as PacBio, Oxford Nanopore, or even reference genomes obtained from Sanger-based platforms, are preferred whenever possible.

We have herewith presented the TargeTALE, a webserver for the identification of TALE genes in Xanthomonas genomes and their many respective potential target plant promoteromes. The tool might be particularly useful for researchers without a bioinformatics background who want to characterize genes targeted by specific strains, especially newly sequenced genomes. The information provided by TargeTALE might also be applied in comparative studies aimed at a better understanding of the adaptation of the pathogen based on the differential selection of target genes in the host and might facilitate future studies in pathogenomics. Finally, with the incorporation of new datasets of promoters from other hosts, these platforms might also be extended to other Xanthomonas species. TargeTALE is available online at the URL shown below and its source code is available at the GitHub repository. 


\section{Author-Recommended Internet Resources}

Apache HTTP server: https://httpd.apache.org

BioPython: http://biopython.org

Bioservices: https://github.com/cokelaer/bioservices

EBI Expression Atlas database: https://www.ebi.ac.uk/gxa

Ensembl database: https://www.ensembl.org

Joint Genome Institute website: https://jgi.doe.gov

GitHub Requests page: https://github.com/requests/requests

GitHub repository: https://github.com/biopro/targetale

TAIR website: https://www.arabidopsis.org

TargeTALE: http://bioprolab.ufpel.edu.br/targeTALE

Uniprot database: http://www.uniprot.org

\section{Literature Cited}

Binns, D., Dimmer, E., Huntley, R., Barrell, D., O'Donovan, C., and Apweiler, R. 2009. QuickGO: A web-based tool for Gene Ontology searching. Bioinformatics 25: 3045-3046.

Boch, J., Scholze, H., Schornack, S., Landgraf, A., Hahn, S., Kay, S., Lahaye, T., Nickstadt, A., and Bonas, U. 2009. Breaking the code of DNA binding specificity of TAL-Type III effectors. Science 326:1509-1512.

Buell, C. R. 2002. Interactions between Xanthomonas species and Arabidopsis thaliana. Page e0031 in: Arabidopsis Book. BioOne Complete. Washington, D.C.

Doyle, E. L., Booher, N. J., Standage, D. S., Voytas, D. F., Brendel, V. P., Vandyk, J. K., and Bogdanove, A. J. 2012. TAL Effector-Nucleotide Targeter (TALE-NT) 2.0: Tools for TAL effector design and target prediction. Nucleic Acids Res. 40 (W1):W117-W122.

Fitzgerald, T. L., Powell, J. J., Schneebeli, K., Hsia, M. M., Gardiner, D. M., Bragg, J. N., McIntyre, C. L., Manners, J. M., Ayliffe, M., Watt, M., Vogel, J. P., Henry, R. J., and Kazan, K. 2015. Brachypodium as an emerging model for cerealpathogen interactions. Ann. Bot. 115:717-731.

Grau, J., Reschke, M., Erkes, A., Streubel, J., Morgan, R. D., Wilson, G. G., Koebnik, R., and Boch, J. 2016. AnnoTALE: Bioinformatics tools for identification, annotation, and nomenclature of TALEs from Xanthomonas genomic sequences. Sci. Rep. 6:21077.

Grau, J., Wolf, A., Reschke, M., Bonas, U., Posch, S., and Boch, J. 2013. Computational predictions provide insights into the biology of TAL effector target sites. PLOS Comput. Biol. 9:e1002962.

Morris, R. T., O'Connor, T. R., and Wyrick, J. J. 2008. Osiris: An integrated promoter database for Oryza sativa L. Bioinformatics 24:2915-2917.

Moscou, M. J., and Bogdanove, A. J. 2009. A simple cipher governs DNA recognition by TAL effectors. Science 326:1501-1501.
Muñoz Bodnar, A., Bernal, A., Szurek, B., and López, C. E. 2013. Tell me a tale of TALEs. Mol. Biotechnol. 53:228-235.

Niño-Liu, D. O., Ronald, P. C., and Bogdanove, A. J. 2006. Xanthomonas oryzae pathovars: Model pathogens of a model crop. Mol. Plant Pathol. 7:303-324.

Peng, Z., Hu, Y., Xie, J., Potnis, N., Akhunova, A., Jones, J., Liu, Z., White, F. F., and Liu, S. 2016. Long read and single molecule DNA sequencing simplifies genome assembly and TAL effector gene analysis of Xanthomonas translucens. BMC Genomics 17:21.

Pérez-Quintero, A. L., Lamy, L., Zarate, C. A., Cunnac, S., Doyle, E., Bogdanove, A., Szurek, B., and Dereeper, A. 2018. daTALbase: A database for genomic and transcriptomic data related to TAL effectors. Mol. Plant-Microbe Interact. 31: 471-480.

Pérez-Quintero, A. L., Rodriguez-R, L. M., Dereeper, A., López, C., Koebnik, R., Szurek, B., and Cunnac, S. 2013. An improved method for TAL effectors DNAbinding sites prediction reveals functional convergence in TAL repertoires of Xanthomonas oryzae strains. PLoS One 8:e68464.

Rhee, S. Y., Beavis, W., Berardini, T. Z., Chen, G., Dixon, D., Doyle, A., GarciaHernandez, M., Huala, E., Lander, G., Montoya, M., Miller, N., Mueller, L. A., Mundodi, S., Reiser, L., Tacklind, J., Weems, D. C., Wu, Y., Xu, I., Yoo, D., Yoon, J., and Zhang, P. 2003. The Arabidopsis Information Resource (TAIR): A model organism database providing a centralized, curated gateway to Arabidopsis biology, research materials and community. Nucleic Acids Res. 31:224-228.

Streubel, J., Baum, H., Grau, J., Stuttmann, J., and Boch, J. 2017. Dissection of TALE-dependent gene activation reveals that they induce transcription cooperatively and in both orientations. PLOS One 12:e0173580.

Vauterin, L., Rademaker, J., and Swings, J. 2000. Synopsis on the taxonomy of the genus xanthomonas. Phytopathology 90:677-682. 